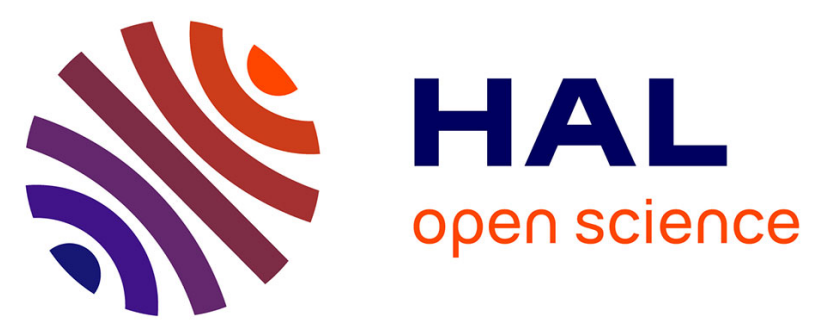

\title{
Pressure-Induced Conversion of a Paramagnetic FeCo Complex into a Molecular Magnetic Switch with Tuneable Hysteresis
}

Yanling Li, Amina Benchohra, Buqin Xu, Benoît Baptiste, Keevin Béneut, Paraskevas Parisiades, Ludovic Delbes, Alain Soyer, Kamel Boukheddaden, Rodrigue Lescouëzec

\section{To cite this version:}

Yanling Li, Amina Benchohra, Buqin Xu, Benoît Baptiste, Keevin Béneut, et al.. Pressure-Induced Conversion of a Paramagnetic FeCo Complex into a Molecular Magnetic Switch with Tuneable Hysteresis. Angewandte Chemie, 2020, 132 (39), p. 17425-17429. 10.1002/ange.202008051 . hal-02947817

\section{HAL Id: hal-02947817 https://hal.sorbonne-universite.fr/hal-02947817}

Submitted on 24 Sep 2020

HAL is a multi-disciplinary open access archive for the deposit and dissemination of scientific research documents, whether they are published or not. The documents may come from teaching and research institutions in France or abroad, or from public or private research centers.
L'archive ouverte pluridisciplinaire HAL, est destinée au dépôt et à la diffusion de documents scientifiques de niveau recherche, publiés ou non, émanant des établissements d'enseignement et de recherche français ou étrangers, des laboratoires publics ou privés. 


\title{
Pressure-Induced Conversion of a Paramagnetic FeCo Complex into a Molecular Magnetic Switch with tuneable hysteresis
}

\author{
Yanling Li, ${ }^{[\mathrm{a}] \star}$ Amina Benchohra,${ }^{[\mathrm{a}]}$ Buqin $\mathrm{Xu},{ }^{[\mathrm{a}]}$ Benoît Baptiste,${ }^{[\mathrm{b}]}$ Keevin Béneut, ${ }^{[\mathrm{b}]}$ Paraskevas \\ Parisiades, ${ }^{[\mathrm{b}]}$ Ludovic Delbes,${ }^{[\mathrm{b}]}$ Alain Soyer, ${ }^{[\mathrm{b}]}$ Kamel Boukheddaden, ${ }^{[\mathrm{c}]}$ Rodrigue Lescouëzec ${ }^{[\mathrm{a}] \star}$
}

\begin{abstract}
A key challenge in the design of magnetic molecular switches is to obtain bistability at room temperature. Here, we show that a moderate pressure allows converting a paramagnetic $\mathrm{Fe}^{\prime \prime \prime}{ }_{2} \mathrm{Co}_{2}{ }_{2}$ square complex into a molecular switch exhibiting a full dia- to para-magnetic transition: $\mathrm{Fe}^{\prime \prime} \mathrm{Co}{ }^{\prime \prime \prime} \Leftrightarrow \mathrm{Fe}{ }^{\text {III }} \mathrm{Co}$ ". Moreover, the complex follows a rare behavior: the higher the pressure is, the broader the magnetic hysteresis is. Thus, the application of an adequate pressure allows inducing a magnetic bi-stability at room temperature with predictable hysteresis width. The structural studies at different pressures suggest that the pressure-enhanced bistability is due to the strengthening of intermolecular interactions upon pressure increase. An original microscopic Ising-like model including pressure effects, is developed to simulate this unprecedented behavior. Overall, this study shows that FeCo complexes could be very sensitive piezo-switches with potential use as sensors.
\end{abstract}

Molecular magnetic switches undergoing drastic changes in their optical, magnetic, dielectric or mechanical properties focus strong interest for their potential use in the manufacture of molecule-based sensors, actuators and memory devices. ${ }^{[1,2]}$ In these molecular solids, external stimuli such as light, temperature, pressure can reversibly switch the electronic and structural properties between two well characterized states. The spin-crossover (SCO) complexes, in particular those with octahedral Fe" $\mathrm{N}_{6}$ coordination sphere, are among the most studied molecular switchable systems. ${ }^{[3]}$ Here, the conversion occurring between a diamagnetic lowspin state and the paramagnetic high-spin state is accompanied by a significant volume change and it is generally sensitive to pressure. This feature makes these systems useful as barocaloric materials for refrigeration technique ${ }^{[4]}$ or as pressure sensors. In fact, the application of a hydrostatic pressure on a SCO compound stabilizes the low-spin state due to its smaller ionic radius and shifts the transition temperature to the high-spin state, $T_{1 / 2}$, upward. ${ }^{[5]}$

\footnotetext{
[a] Y. Li, A. Benchohra, S. De, R. Lescouëzec

Institut Parisien de Chimie Moléculaire, CNRS UMR 8232 Sorbonne Université

4 place Jussieu 75252 Paris cedex 5

E-mail: yanling.li@sorbonne-universite.fr,

rodrigue.lescouezec@sorbonne-universite.fr

[b] K Beneut, P. Parisiadis, B. Baptiste, L. Delbes, A. Soyer Institut de Mineralogie, de Physique des Materiaux et de Cosmochimie (IMPMC), Sorbonne Universite, UMR 7590 CNRS, UMR 206 IRD, Museum National d'Histoire Naturelle MNHN, 4 place Jussieu, F-75005 Paris, France.

[b] K. Boukheddaden Université Paris-Saclay, UVSQ, Groupe d'Etudes de la Matière Condensée, CNRS UMR No 8635, 45 Avenue des Etats-Unis 78035 , Versailles cedex.
}

Supporting information for this article is given via a link at the end of the document.
The pressure can also significantly affect the cooperativity of SCO transition by modifying the crystal packing of SCO compounds, hence the elastic energy of the systems. In a more chemical description, changes in the intermolecular interactions should affect the cooperativity of the system. In most of the cases, the pressure induces a decrease of cooperativity: the thermal hysteresis shrinks or the transition becomes less steep. ${ }^{[6]}$ Any other behaviour, such as an increase of the thermal hysteresis or a non-monotonous behaviour under pressure, is denoted as anomalous. ${ }^{[7]}$

The cyanide-bridged FeCo complexes showing metal-metal Electron Transfer Coupled to a Spin Transition (ETCST) are another class of magnetic switches that share many features with $\mathrm{Fe}^{\|} \mathrm{SCO}$ complexes. ${ }^{[8-10]}$ Here the electronic configuration switches between a $\left\{\mathrm{Fe}^{\prime \prime}{ }_{\mathrm{LS}}-\mathrm{Co}{ }^{\prime \prime \prime}{ }_{\mathrm{LS}}\right\}$ diamagnetic state and a \{Fe ${ }^{\text {III }} \mathrm{LS}^{-\mathrm{CO}}{ }_{\mathrm{HS}}$ \} paramagnetic one (Fig. 1a). This situation can occur as the energy difference, $\Delta \mathrm{E}$, between both states is small enough and can be reversed by application of stimuli. In these switches, the structural changes arise from the Co-ligand bond variations and are similar to those found in $\mathrm{Fe}$ " SCO complexes. These systems could thus act as piezo-switches, the high pressure stabilizing the more contracted $\left\{\mathrm{Fe}^{\prime \prime} \mathrm{LS}^{-} \mathrm{CO}{ }^{\text {III }} \mathrm{LS}\right\}$ diamagnetic state. To our knowledge, there is only one report investigating the influence of pressure on the ETCST transition in low dimensional molecular system. In fact, Li et coll. recently studied a $\left\{\mathrm{Fe}_{2} \mathrm{CO}_{2}\right\}$ square complex showing a gradual ETCST transition near $200 \mathrm{~K}^{[11]}$ In this case, the incomplete transition becomes more gradual under pressure, following thus the common behaviour described in many $\mathrm{Fe}^{\|}$SCO complexes. In the present work we decided to further explore the potentialities of these FeCo charge transfer molecules as piezo-switches.

We selected for this study the paramagnetic compound of formula $\quad\left\{\left[\mathrm{Fe}(\mathrm{Tp})(\mathrm{CN})_{3}\right]_{2}\left[\mathrm{Co}(\text { vbik })_{2}\right]_{2}\right\}^{2+} \quad(\mathrm{Tp}=$ hydrotris (pyrazolyl)borate; vbik = bis(1-vinylimidazol-2-yl)ketone) for the reasons described below. As reported in a previous study, this complex exhibits a thermally induced ETCST near room temperature in solution. ${ }^{[12]}$ This permits to crystallize it either in its $\left\{\mathrm{Fe}^{\mathrm{III}}{ }_{2} \mathrm{Co}_{2}\right\}^{2+}$ paramagnetic state when the temperature is set at $35^{\circ} \mathrm{C}$ (phase 1 ) or in its $\left\{\mathrm{Fe}_{2}{ }_{2} \mathrm{Co}^{\mathrm{III}}{ }_{2}\right\}^{2+}$ diamagnetic state when temperature is set at $5^{\circ} \mathrm{C}$ (phase 2). While the phase 2, $\left\{\left[\mathrm{Fe}(\mathrm{Tp})(\mathrm{CN})_{3}\right]_{2}\left[\mathrm{Co}\left(\mathrm{vbik}_{2}\right]_{2}\right\}\left(\mathrm{BF}_{4}\right)_{2} \bullet 12 \mathrm{H}_{2} \mathrm{O}, \mathrm{MeOH}\right.$, shows a steep thermally-induced ETCST between ca. 320-350 K, the solvatomorph 1, $\left\{\left[\mathrm{Fe}(\mathrm{Tp})(\mathrm{CN})_{3}\right]_{2}\left[\mathrm{Co}(\text { vbik })_{2}\right]_{2}\right\}\left(\mathrm{BF}_{4}\right)_{2} \cdot 2 \mathrm{MeOH}$, is trapped in a paramagnetic state. ${ }^{[12]}$ These differences underline the impact of the solid-state interactions on the ETCST phenomenon, as also reported in recent works. ${ }^{[13,14]}$ The existence of a thermally-induced ETCST in solution and in the crystal phase 2 indicates that the diamagnetic and paramagnetic states are close in energy in the $\left\{\left[\mathrm{Fe}(\mathrm{Tp})(\mathrm{CN})_{3}\right]_{2}\left[\mathrm{Co}(\mathrm{vbik})_{2}\right]_{2}\right\}^{2+}$ square complex (Figure 1b). We thus postulated that pressure should allow recovering the transition behaviour in the solid state in 1. This is actually the case as the investigated complex shows an interesting anomalous behaviour: upon pressure, the 
magnetic bi-stability can be introduced near room temperature. In this work, we thus study the electronic state of the square complex under hydrostatic pressure, by using magnetic susceptibility measurement, micro-Raman spectroscopy, and high-pressure single crystal X-Ray Diffraction experiments. We also simulated the ETCST hysteresis width by using a microscopic Ising-like model. The overall magneto-structural correlation allows suggesting the probable origin of the unusual pressure-enhanced cooperativity. (a)

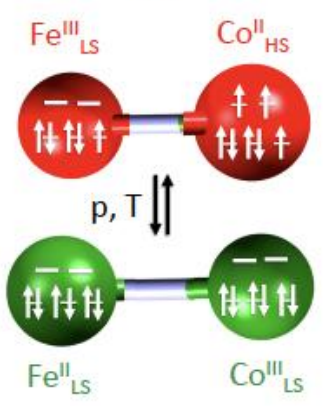

(c)

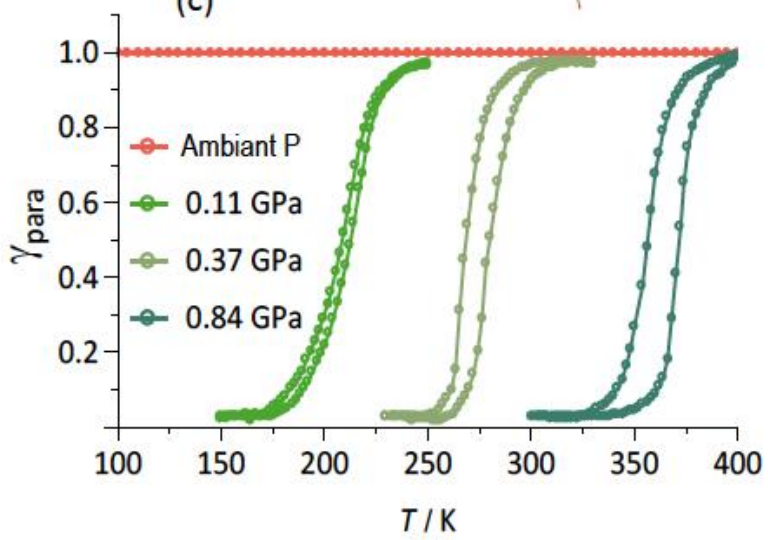

Figure 1. (a) Scheme of the electronic state changes occurring in the Fe-CN-Co pair. (b) Perspective view of the square complex in $\mathbf{1}$ at $10^{-5}$ and $0.85 \mathrm{GPa}$. (c) Thermal variation of the paramagnetic $\left\{\mathrm{Fe}_{2}{ }_{2} \mathrm{Co}_{2}{ }_{2}\right\}$ molar fraction $\gamma_{\text {para }}$ in $\mathbf{1}$ under various hydrostatic pressures.

Figure 1c shows the temperature dependence of the paramagnetic molar fraction in 1, $\gamma_{\text {para, }}$ (deduced from the magnetic susceptibility; see $\mathrm{SI}$ ) under three hydrostatic pressures. At ambient pressure $\mathbf{1}$ is paramagnetic in the whole temperature range. The measured $\chi_{M} T$ value, ca. $7.9 \mathrm{~cm}^{3} \mathrm{~mol}^{-1} \mathrm{~K}$ $X_{M}$ is the molar magnetic susceptibility per $\left\{\mathrm{Fe}_{2} \mathrm{Co}_{2}\right\}$ formula unit) is coherent with the occurrence of isolated paramagnetic ions: two $\mathrm{Fe}$ "III LS and two Co ${ }_{\text {HS. }}{ }^{[15]}$ As expected, under hydrostatic pressure, 1 becomes diamagnetic at low temperature. The pressure threshold for the intra-molecular electron transfer is between 0.05 and $0.11 \mathrm{GPa}$. The piezo-induced electron transfer seems complete as the residual paramagnetic signal is no more measurable. It is also fully reversible as 1 returns to the initial paramagnetic state as the pressure is released. Upon heating, 1 exhibits a thermally induced ETCST transition, whose transition temperature increases with the applied pressure (Fig. 1c). As expected, the hydrostatic pressure favours the diamagnetic state, which is stabilized over a larger temperature range as the pressure increases. Such behaviour was already observed in some cyanide-bridged inorganic polymers,
$\mathrm{ACo}_{4}\left[\mathrm{Fe}(\mathrm{CN})_{6}\right]$, also called Prussian blue analogues (PBA). ${ }^{[16]}$ However, in the present case, the pressure-induced ETCST transition exhibits a hysteretic behavior. These thermal hysteresis loops are reproducible (Fig. S4), and remarkably, their width increases when the pressure increases (Fig. 1). Such anomalous behavior is unprecedented for $\mathrm{FeCo}$ charge transfer systems.

The figure $2 \mathrm{a}$ shows the linear relationship between the transition temperatures measured upon heating and cooling, $T_{1 / 2} \uparrow$ and $T_{1 / 2} \downarrow$, versus pressure. As observed in SCO complexes, this behaviour agrees with the Clapeyron's law:

$$
T_{1 / 2}(p)=T_{1 / 2}(0)-\left(p-p_{0}\right) \frac{\Delta V}{\Delta S},
$$

where, $\Delta V$ (negative) is the volume change between diamagnetic and paramagnetic states, and $T_{1 / 2}(0)$ is the transition temperature corresponding to the threshold pressure value, $p_{0}$, beyond which ETCST transition takes place. Interestingly, the measured shift rates of $T_{1 / 2} \uparrow$ and $T_{1 / 2} \downarrow, 228.4$ and $213.6 \mathrm{~K} \mathrm{GPa}^{-1}$, respectively, they are larger than those measured in related $\mathrm{FeCo} P B A, \mathrm{~K}_{0.1} \mathrm{Co}_{4}\left[\mathrm{Fe}(\mathrm{CN})_{6}\right]_{2.7} \cdot 18 \mathrm{H}_{2} \mathrm{O},(170$ $\left.\mathrm{K} \mathrm{GPa}^{-1}\right)^{[16]}$ and other SCO solids $\left(190-200 \mathrm{~K} \mathrm{GPa}^{-1}\right){ }^{[17]}$ The present square molecule is thus more sensitive to pressure than the corresponding three-dimensional inorganic polymers. The linear relation between $T_{1 / 2}$ and $p$ allows estimating the pressure threshold values, $p_{1 / 2} \uparrow=0.55 \mathrm{GPa}$ and $p_{1 / 2} \downarrow=0.5 \mathrm{GPa}$, that should promote the transition at $300 \mathrm{~K}$, in isothermal conditions (by drawing a horizontal line at $300 \mathrm{~K}$ ). These data are in full agreement with the pressure-dependent Raman spectra of 1 that allow monitoring the electronic state of the molecule by probing the $v_{\mathrm{CN}}$ stretching vibration (see $\mathrm{SI}$ ). Importantly, the linear increase of the width of the thermal hysteresis loop under pressure, of ca. $15 \mathrm{~K}$ per GPa, indicates an enhancement of the cooperative elastic interactions with pressure. Considering the similarity between SCO and ETCST systems, this anomalous transition was rationalized in a phenomenological approach by using a microscopic Ising-like mechano-elastic model, which has been successfully used to model various types of SCO systems ${ }^{[18]}$ but never used yet for ETCST transition. This simple model is based on the description of the $\mathrm{Fe}_{2} \mathrm{Co}_{2}$ square by a two-states fictitious spin, $s$ whose eigenvalues -1 , and +1 are respectively associated with the diamagnetic Fe III $_{\text {LS }}$ - CO"HS, and the paramagnetic Fe" ${ }_{\text {LS }}-\mathrm{CO}^{\text {III }}$ LS states. These states have different degeneracies, respectively denoted, $g_{\text {dia }}$ and $g_{\text {para }}$ with as $g_{\text {para }}>>g_{\text {dia }}$, due to the significant difference of their electronic (spin state) and vibrational (density of states) properties. At $0 \mathrm{~K}$, the fundamental diamagnetic state and the excited paramagnetic state are separated by the energy barrier, $\Delta_{0}=E_{\text {para }}-E_{d i a}$, In practice, this energy difference is correlated to the differences in redox potential between the $\mathrm{Fe}^{\text {III/I }}$ and $\mathrm{Co}^{\mathrm{III/I}}$ couples. ${ }^{[19]}$ The diamagnetic/paramagnetic transition can thus be described using an Ising-like model, whose Hamiltonian is:

$H=-J_{0} \sum_{i} s_{i} s_{j}+\Delta_{\mathrm{eff}} \sum_{i} s_{i}$.

Here $J_{0}>0$ stands for the ferroelastic interaction and the effective energy gap, $\Delta_{\text {eff }}=\Delta_{0}-k_{B} T \ln g$, contains enthalpic and entropic contribution, $k_{B} \ln \left(g_{\mathrm{para}} / g_{\mathrm{dia}},\right)$. In this model, the expression of the transition temperature is easily obtained as $T_{1 / 2}^{0}=\frac{\Delta_{0}}{k_{B} \ln g}$. The phase diagram of the present model is simple: (i) a first-order phase transition with a thermal hysteresis is obtained when $T_{C}^{0}>T_{1 / 2}^{0}$, where $T_{C}^{0}=\frac{z J_{0}}{k_{B}}$ where $z$ is the lattice 
coordination number (ii) otherwise a gradual conversion takes place (see SI). The application of an external pressure, $p$, on the lattice affects both the energy barrier, $\Delta_{0}$, and the elastic interaction $J_{0}$, whose expressions become:

$$
\Delta=\Delta_{0}+\alpha \times P \text { and } J=z J_{0}+\gamma \times p, \text { (3) }
$$

Following the standard resolution of Ising-like models in meanfield approximation (see $\mathrm{SI}$ ), it is possible to express the fraction of paramagnetic state as a function of temperature. According to $\alpha, \gamma, T_{1 / 2}^{0}$ and $T_{C}^{0}$ values, several behaviors are possible, among them, the present experimental case showing an increase of the thermal hysteresis width (cooperativity) as well as the transition temperature under pressure. This occurs when $\alpha$ and $\gamma$ are taken strictly positive, which means that the pressure strengthens both the energy difference, $\Delta=E_{\text {para }}-E_{\text {dia }}$ (stabilizing the diamagnetic one with a smaller volume) and the long-range elastic interactions $(J)$ in the system.

In order to bring further evidence of the piezo-induced ETCST and to enlighten the possible origin of the unusual pressureenhanced bistability, single-crystal X-ray diffraction experiments were carried out at $300 \mathrm{~K}$ under several pressures up to 1.20 $\mathrm{GPa}$. While the quality of the structural model at $1.20 \mathrm{GPa}$ is too moderate to be fully exploited, relevant structural data of the metal coordination spheres were obtained at $0.12,0.37,0.71$ and $0.85 \mathrm{GPa}$ (details in $\mathrm{SI}$ ). Although the space group P-1 is conserved up to the maximum applied pressure, significant changes occur in the molecular geometry, which are consistent with a $\mathrm{Fe}^{\prime \prime \prime \prime}{ }_{L S} \mathrm{CO}_{\mathrm{HS}}=>\mathrm{Fe}^{\prime \prime}{ }_{\mathrm{LS}} \mathrm{Co}^{\text {III }} \mathrm{LS}$ conversion. In particular, the geometry of the Co coordination sphere is particularly affected by the ETCST. First of all, while the average length of the Co-N coordination bonds is about $2.1 \AA$ in the range $0.00-0.37 \mathrm{GPa}$, it drops to about $1.9 \AA$ above $0.71 \mathrm{GPa}$ (Fig. S6). These values are typical of high-spin Co" and low-spin Co"l' complexes, respectively. The transition thus takes place at $300 \mathrm{~K}$ in the pressure range of $0.37-0.71 \mathrm{GPa}$. This agrees with the results obtained by Raman spectroscopy (see SI) and magnetic measurements. Secondly, the distortion of cobalt coordination sphere is also indicative of the Co redox state. The total deviation from orthogonality of the twelve $\mathrm{N}-\mathrm{Co}-\mathrm{N}$ angles, $\Sigma$, varies slightly around $45^{\circ}$ in the pressure range, $0.00-0.37 \mathrm{GPa}$, which agrees with the occurrence of a $\mathrm{Co}$ (II) high-spin state (Fig. S7). However, it drops to $16-20^{\circ}$ in Co' low-spin state at higher pressures (Fig. S7). The situation is different for the $\left\{\mathrm{Fe}(\mathrm{Tp})(\mathrm{CN})_{3}\right\}$ sub-unit. As previously observed, in photo-induced ETCST, ${ }^{[20]}$ there are no significant differences between the geometry of the low spin $\mathrm{Fe}^{\prime \prime} \mathrm{C}_{3} \mathrm{~N}_{3}$ coordination sphere (measured here at $0.85 \mathrm{GPa}$ ) and that of the low-spin $\mathrm{Fe}^{\text {III }} \mathrm{C}_{3} \mathrm{~N}_{3}$ (measured at $0 \mathrm{GPa}$ ).
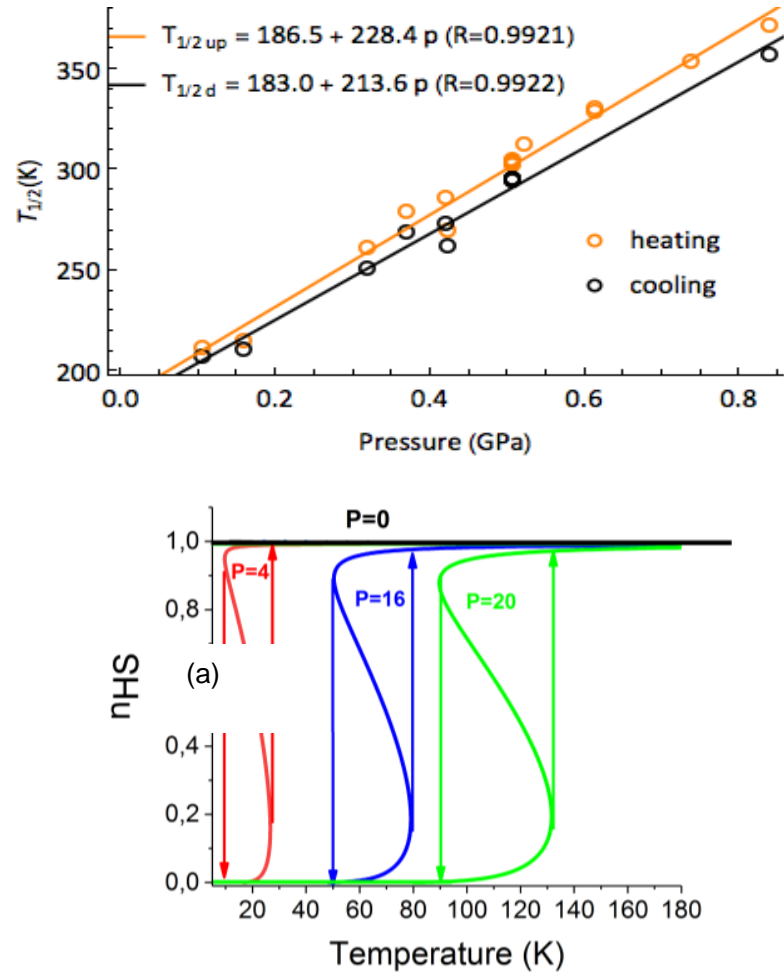

Figure 2. (a) Pressure dependence of the ETCST transition temperatures heating and cooling $T_{1 / 2}$. (b) Thermal dependence of the paramagnetic fraction under pressure showing a transformation from a strict paramagnetic state to first-order spin transition as the pressure increases from 0 to $20 \mathrm{kbar}$ in line witl (b) :xperimental results. The model parameter values are: $\sqrt{g}=$ $z J_{0}=120 \mathrm{~K}, \alpha=200 \mathrm{~K} / \mathrm{GPa}, \gamma=80 \mathrm{~K}^{\mathrm{GPPa}}{ }^{-1}$.

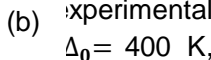



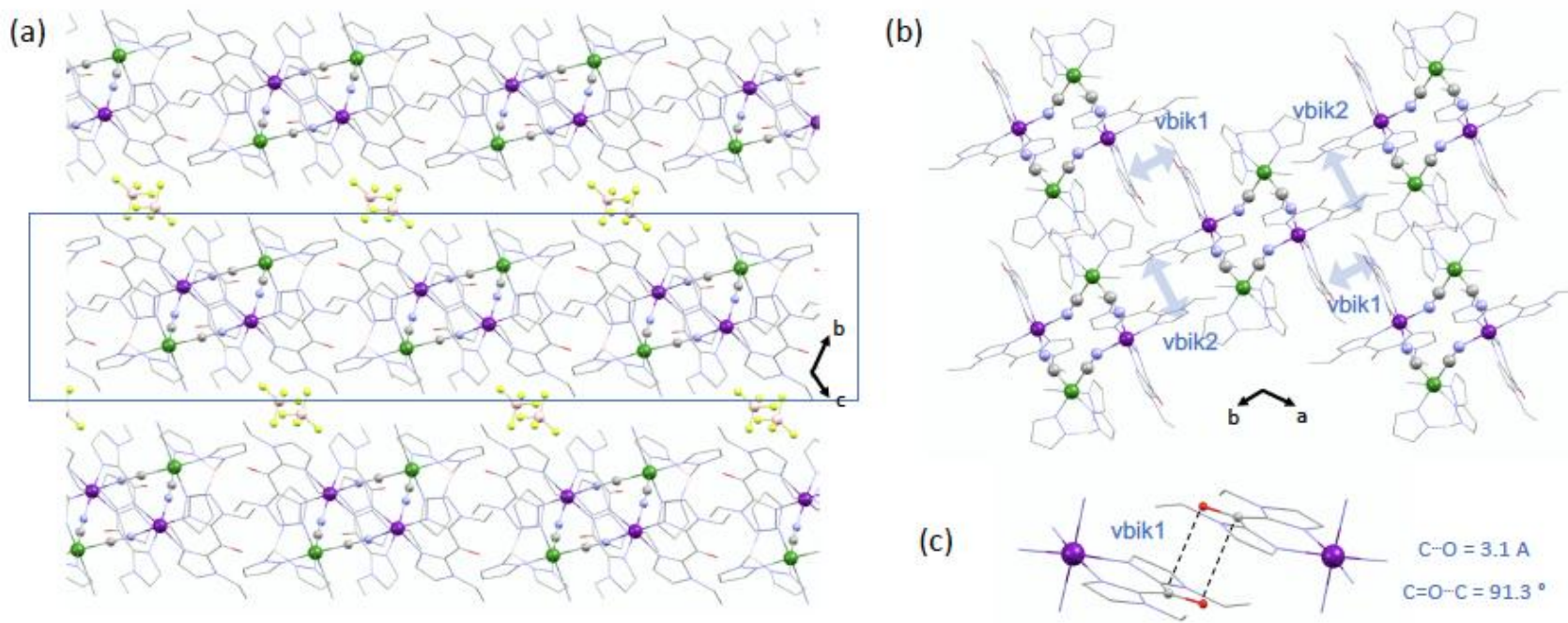

Figure 3. (a) Perspective view of the supramolecular sheet of square complexes separated by $\mathrm{BF}_{4}$ anions; (b) Perspective view showing the intermolecular interaction involving the vbik ligands. (c) Perspective view of the dipolar interaction between the $\mathrm{CO}$ groups belonging to neighbouring vbik1 ligands. (Co: violet, Fe: green, C: grey, $\mathrm{N}$ : light blue, O: red, F: yellow, B: pink)

The changes of intermolecular interactions and short contact distances under pressure were carefully analysed to determine the possible origin of the pressure-enhanced cooperativity. First of all, it is worth noticing that the pressure does not significantly modify the crystal packing, which can be described as cationic sheets of square complexes aligned parallel to the $(a, b-c)$ plan and, separated from each other by $\mathrm{BF}_{4}$ counterion (Fig. 3a). In order to get a first evaluation of the pressure effect on the intermolecular interactions, we first listed all the short contacts in 1 at different pressures (SI). It appears that the number of short contacts is higher inside the sheets than between the sheets, whatever the pressure is. The analysis of all these contacts reveals that the square complexes are connected through intermolecular interactions involving the peripheral vbik ligands. Actually, the most striking features of the crystal packing are the two stackings of the vbik1 and of the vbik2 ligands of neighboring square complexes, as showed in the figure $3 \mathrm{~b}$ (and $\mathrm{SI}$ ). This stacking goes along two orthogonal directions and leads to a grid-like supramolecular framework. These interactions are mainly of electrostatic nature as they involve pairs of electronrich and electron-poor atoms of neighboring vbik ligands. Such interactions are not so known as classical $\pi$-interactions but they can show higher stacking energy of ca. $10-20 \mathrm{kcal} / \mathrm{mol} .^{[21]}$ In the case of vbik1, these interactions are combined with an antiparallel arrangement of two $\mathrm{C}=\mathrm{O}$ groups in close contact, which build an additional dipolar interaction whose energy values are similar to those of $\mathrm{H}$-bonds. ${ }^{[21]}$ Upon pressure, as the distances decrease (see $\mathrm{SI}$ ), one would expect that the dominant electrostatic interactions are reinforced, leading to the enhancement of the elastic interactions that is responsible for the unusual pressure-induced bistability observed in $\mathbf{1}$.

In conclusion, we showed that the application of pressure allows recovering the thermally-induced charge transfer in a FeCo complex that shows ETCST in solution but which is trapped in its paramagnetic state in the solid-state. Remarkably, the pressureinduced $\mathrm{Fe}^{\text {III } \mathrm{Co}}$ " $\Leftrightarrow \mathrm{Fe}^{\prime \prime} \mathrm{Co}^{\prime \prime \prime}$ conversion is reversible and complete even at a pressure as low as $0.11 \mathrm{GPa}$. More importantly, the complex displays an unprecedented behavior for charge transfer switchable complexes: a hysteresis broadening upon increasing pressure. A theoretical Ising model directly inspired from works on SCO complexes, allows rationalizing this phenomenon, and suggests an enhancement of both the elastic interactions and $E_{\text {para }}-E_{\text {dia }}$ energy difference. The advanced $\mathrm{XRD}$ measurements at five pressures reveal that the $\mathrm{Fe}_{2} \mathrm{Co}_{2}$ squares are closely linked to each other by electrostatic interactions to form a supramolecular 2D network. The increase of intermolecular interactions mediated by the vbik ligand is probably responsible for an enhancement of the elastic interactions between the units, and for the pressure- enhanced bistability. Overall, these properties make this compound a good candidate as temperature and pressure sensor. Additional advanced XRD experiments under pressure at different temperatures and the study of derivative complexes with other counter-ions should help confirming the origin of this unusual behaviour.

\section{Acknowledgements}

We would like to thank Philippe Rosier and Nicolas Dumesnil (from the Cellule Projet of IMPMC) for the production of mechanical parts which were useful for mounting the diamond anvil cell on the diffractometer, Frederic Datchi and Yoann Guarnelli (IMPMC) for the preparation of the diamond anvil cell, and Pierre Fertey (CRISTAL beamline, SOLEIL) for his help during the high pressure $X$-ray diffraction experiments (proposal 20170382).

\section{[1] K. Senthil Kumar, M. Ruben, Coordination Chemistry} Reviews 2017, 346, 176-205.

[2] G. Molnár, S. Rat, L. Salmon, W. Nicolazzi, A. Bousseksou, Advanced Materials 2018, 30, 1703862.

[3] M. A. Halcrow, Ed. , Spin-Crossover Materials: Properties and Applications, Wiley, Chichester, 2013.

[4] S. P. Vallone, A. N. Tantillo, A. M. dos Santos, J. J. Molaison, R. Kulmaczewski, A. Chapoy, P. Ahmadi, M. A. Halcrow, K. G. Sandeman, Adv. Mater. 2019, 31, 1807334. 
[5] P. Gutlich, V. Ksenofontov, A. Gaspar, Coordination Chemistry Reviews 2005, 249, 1811-1829.

[6] P. Gütlich, V. Ksenofontov, A. B. Gaspar, Coordination Chemistry Reviews 2005, 249, 1811-1829.

[7] A. B. Gaspar, G. Molnár, A. Rotaru, H. J. Shepherd, Comptes Rendus Chimie 2018, 21, 1095-1120.

[8] Y.-S. Meng, O. Sato, T. Liu, Angewandte Chemie International Edition 2018, 57, 12216-12226.

[9] C. Mathonière, European Journal of Inorganic Chemistry 2018, 2018, 248-258.

[10] G. N. Newton, M. Nihei, H. Oshio, European Journal of Inorganic Chemistry 2011, 2011, 3031-3042.

[11] L. Cao, J. Tao, Q. Gao, T. Liu, Z. Xia, D. Li, Chem Commun. 2014, 50, 1665-1667.

[12] S. De, J.-R. Jiménez, Y. Li, L.-M. Chamoreau, A. Flambard, Y. Journaux, A. Bousseksou, R. Lescouëzec, RSC Advances 2016, 6, 17456-17459.

[13] C.-Q. Jiao, W.-J. Jiang, Y.-S. Meng, W. Wen, L. Zhao, J.-L. Wang, J.-X. Hu, G. G. Gurzadyan, C.-Y. Duan, T. Liu, National Science Review 2018, 5, 507-515.

[14] M. Nihei, Y. Yanai, I.-J. Hsu, Y. Sekine, H. Oshio, Angew. Chem. Int. Ed. 2017, 56, 591-594.

[15] F. Lloret, M. Julve, J. Cano, R. Ruiz-García, E. Pardo, Inorganica Chimica Acta 2008, 361, 3432-3445.

[16] A. Bleuzen, J.-D. Cafun, A. Bachschmidt, M. Verdaguer, P. Münsch, F. Baudelet, J.-P. Itié, J. Phys. Chem. C 2008, 112, 17709-17715.

[17] P. Gutlich, V. Ksenofontov, A. Gaspar, Coordination Chemistry Reviews 2005, 249, 1811-1829.

[18] K. Boukheddaden, I. Shteto, B. Hôo, F. Varret, Phys. Rev. B 2000, 62, 14796-14805.

[19] M. Nihei, K. Shiroyanagi, M. Kato, R. Takayama, H. Murakami, Y. Kera, Y. Sekine, H. Oshio, Inorg. Chem. 2019, 58, 11912-11919.

[20] A. Mondal, Y. Li, M. Seuleiman, M. Julve, L. Toupet, M. Buron-Le Cointe, R. Lescouëzec, Journal of the American Chemical Society 2013, 135, 1653-1656.

[21] K. Molčanov, V. Milašinović, B. Kojić-Prodić, Crystal Growth \& Design 2019, 19, 5967-5980. 
